\title{
Congenital Rubella with Autism and Evidence of a Remote Stroke
}

\author{
Jill Hutton ${ }^{1^{*}}$ and George J Hutton ${ }^{2}$ \\ ${ }^{1}$ Department of Obstetrics and Gynecology, The Woman's Hospital of Texas, Houston, Texas, USA \\ ${ }^{2}$ Department of Neurology, Baylor College of Medicine, Houston, Texas, USA
}

"Corresponding author: Jill Hutton, Department of Obstetrics and Gynecology, The Woman's Hospital of Texas, 2617 W. Holcombe C \#285, Houston, Texas 77025, USA, Tel: 281-772-7861; E-mail: jachmd@yahoo.com

Received date: 02 Sep 2014; Accepted date: 24 Oct 2014; Published date: 27 Oct 2014

Copyright: () 2014 Hutton J, et al. This is an open-access article distributed under the terms of the Creative Commons Attribution License, which permits unrestricted use, distribution, and reproduction in any medium, provided the original author and source are credited.

\begin{abstract}
Background: The conventional diagnosis of congenital rubella is a rare occurrence of an obvious maternal exposure, resulting in an infant born with obvious signs such as cataracts, deafness, microcephaly or congenital heart disease.

Patient: In this case, a mother with no known symptom, sign or exposure of rubella gave birth to a male infant without complication. The infant had no notable abnormalities at birth. The boy had developmentally delay, and between the ages of five and seven years was diagnosed with autism. At the age of ten years, an MRI of his brain evidenced a remote stroke. Careful examination of all medical records showed that the mother had a considerable rise in her rubella titer and thus had been exposed to rubella during pregnancy.
\end{abstract}

Result: The diagnosis of congenital rubella was recognized more than ten years after the birth of the infant; congenital rubella is linked to both autism and ischemic brain injury.

Conclusions: This case illustrates how both maternal rubella exposure and congenital rubella are likely underrecognized, and how a newborn may exhibit no outward symptoms at birth, yet the elusive diagnosis of congenital rubella is considered years later.

Keywords: Congenital rubella; Ischemic brain injury; Autism; Stroke

\section{Introduction}

Congenital rubella is thought to be rare in the United States of America (USA) after widespread vaccination protocols were put into place in the late 1960 s to early 1970 s. This rare event is stereotypically associated with a mother, often foreign born and never immunized, who delivers an infant with overt congenital rubella syndrome recognizable at birth, with obvious signs such as cataracts, microcephaly, deafness or congenital heart disease [1]. Less recognizable, would be a mother, USA born and previously vaccinated, who delivers an infant with no obvious signs at birth, yet most likely affected by congenital rubella with autism and evidence of a remote stroke. This case illustrates how both maternal rubella exposure and congenital rubella are probably under-recognized, and how a newborn may exhibit no outward symptoms at birth, yet the elusive diagnosis of congenital rubella is considered years later.

\section{Case Presentation}

In 1999, a male infant was born vaginally at 38 weeks 6 days gestation. The mother was a USA born physician at 30 years of age mostly working at a busy, urban, county hospital with a large immigrant population. The mother had a single rubella vaccination in 1972 (and possibly a booster in 1991, but documentation only gives evidence of a measles vaccine, not measles mumps rubella vaccine [MMR]). She was non-immune to rubella at her first prenatal visit at 10 weeks gestation. The pregnancy was without complication except for an echogenic cardiac focus noted at 19 weeks (Figure 1). At delivery, her rubella screen showed immunity.

The infant was 6 pound 6 ounces, 20 inches long and had a head circumference of 13 inches $/ 33 \mathrm{~cm}(5-10 \%)$. He had normal hearing tests. At 2 months his head circumference was 15.5 inches $/ 39.4 \mathrm{~cm}$ (25-50\%). He met all motor milestones, but was delayed in speech and exhibited social anxiety. At 18 months, he had a single febrile seizure with negative lumbar puncture. At 22 months an electroencephalogram was negative.

Between 5 and 7 years of age, he was diagnosed with autism. His intelligence quotient (IQ) was found to be in the normal range of 100-110. He also demonstrated dysgraphia and difficulties with sensory integration.

At age 10, he underwent functional magnetic resonance imaging (MRI) as part of an elective research study for children with autism. Non-contrast T1-weighted images incidentally revealed a cystic area in the posterior temporal lobe of the brain (Figure 2). This was further investigated with a full brain MRI showing cystic encephalomalacia in the subcortical white matter of the left posterior temporo-parietal occipital lobes and left basal ganglia most consistent with a remote stroke. Anteriorly there were prominent perivascular spaces (Figures $3 \mathrm{a}$ and $3 \mathrm{~b}$ ). A year later, these findings were found to be stable on repeat brain MRI. Carotid and cerebral arteries were without abnormalities on magnetic resonance angiogram (MRA). His neurological exam was normal. Clotting studies including factor $\mathrm{C}$ resistance, factor $\mathrm{C}$ function, factor $\mathrm{S}$ function, antithrombin activity, anticardiolpin $\operatorname{IgG/IgM,~partial~thromboplastin~time-lupus~}$ 
anticoagulant (PTT-LA), dilute Russell viper venom test (DRVVT) lupus anticoagulant and homocysteine were all normal. A transthoracic echocardiogram was also normal. The most likely diagnosis is congenital rubella.

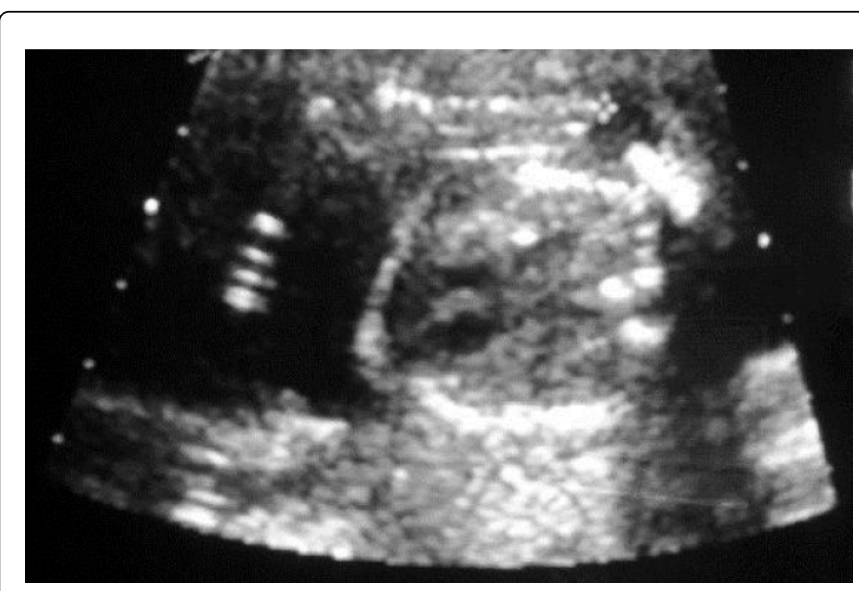

Figure 1: Echogenic cardiac focus, Accuson ultrasound image, from VCR tape, 1999.

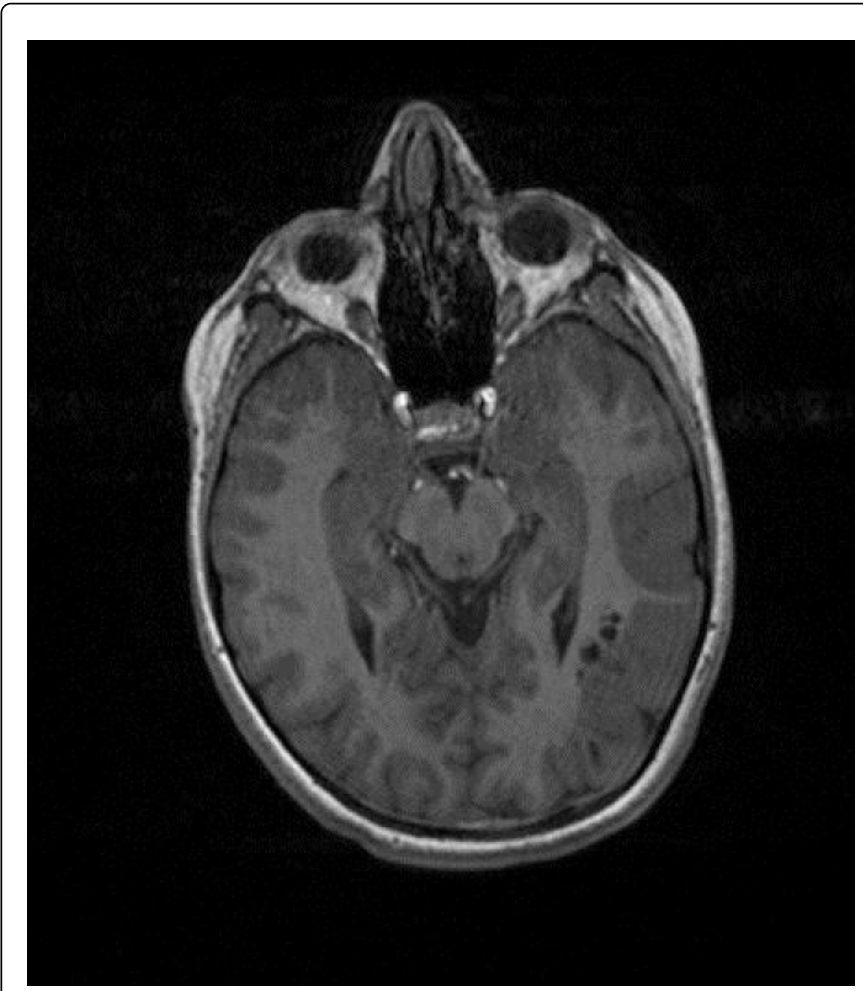

Figure 2: Axial non-contrast T1-weighted MR image, from study 2010.

\section{Discussion}

Congenital rubella is thought to be rare in the United States thanks to widespread vaccination. In this case, the mother, a physician born in the USA, received a single dose of MMR in 1972. A second dose of
MMR was only added to the vaccine schedule in 1989 [2]; therefore, mothers born before this recommendation change may have received only one rubella vaccine and may be more likely to be non-immune. The most common way to diagnose rubella is an increase in rubella titer [3]. The mother presented above was rubella non-immune at 10 weeks gestation, but at the time of delivery her rubella specific IgG was elevated. The mother had no documentation of any symptom, sign or exposure related to rubella. She was not stereotypically foreign born or unvaccinated, although in hindsight had probable exposure while working in a busy county hospital. Such exposure is doubtfully limited to healthcare workers, as countless women have jobs in public service industries. Maternal rubella exposure is likely under-recognized and under-diagnosed due to the fact that most infected individuals have few or no symptoms [3].

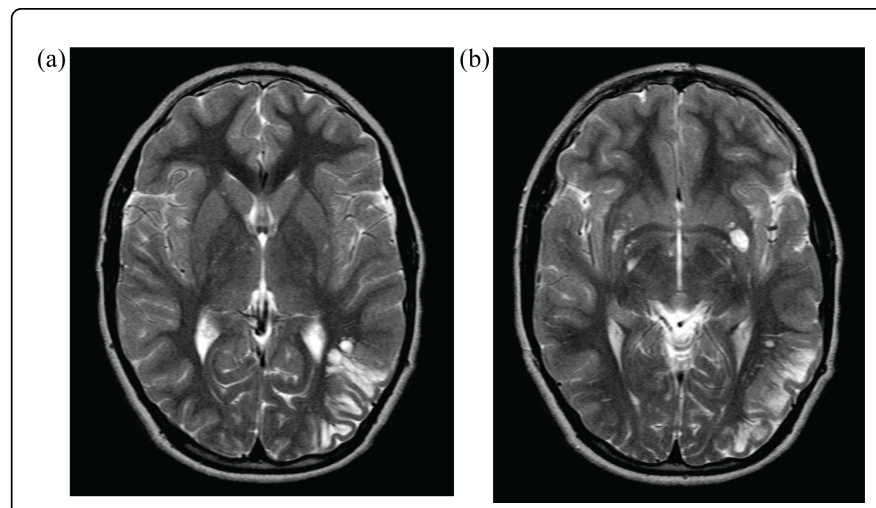

Figure 3: Selected axial T2-weighted MR images, 2010 ( $a$ and b).

A single echogenic cardiac focus, as found in this case by ultrasound at 19 weeks, may indicate inflammation [4]. A study by Hutton et al., mentions two such echogenic cardiac foci found on screening ultrasounds of pregnancies carried by patients who converted from rubella non-immune to immune; these small numbers warrant further investigation [5].

The MRI findings above add to the likelihood of the diagnosis of congenital rubella, especially since other etiologies for a remote stroke were excluded, including cardiac anomalies, clotting dysfunction, and arterial abnormalities. Postmortem studies of brains affected by congenital rubella by Rorke found frequent foci of ischemia and small associated cysts along penetrating vessels deep in the white matter and basal ganglia of brain specimens; microcephaly was also common [6]. An MRI study by Lane et al. of 11 deaf adults who survived congenital rubella showed ischemic sequelae in the deep white matter, periventricular regions and the parietal lobe [7]. It is possible that the brain lesions in our case are from congenital rubella and the autism may be of a separate cause; however, congenital rubella was linked as a cause of autism by Chess in 1971 [8,9]. Autism is indeed a spectrum and rubella may be implicated in certain cases. Congenital rubella should remain a consideration in the differential diagnosis of autism.

Lastly, this study shows how congenital rubella went unrecognized for over ten years in a boy who had no obvious symptoms at birth, though his mother's rubella titer increased during his gestation. Had he not been enrolled in a research study which resulted in an MRI, the diagnosis of rubella may have never been suspected. Evidence of a remote stroke led to multiple tests looking for causes of stroke. As causes of stroke were eliminated, the diagnosis of congenital rubella as 
Citation: Hutton J, Hutton GJ (2014) Congenital Rubella with Autism and Evidence of a Remote Stroke. J Vaccines Vaccin 5: 258. doi:

Page 3 of 3

a unifying diagnosis of autism and stroke became more probable. Accurate data on the incidence of congenital rubella and its burden on society are surely under-appreciated.

\section{References}

1. Berger BE, Omer SB (2010) Could the United States experience rubella outbreaks as a result of vaccine refusal and disease importation? Hum Vaccin 6: 1016-1020.

2. Banatvala JE, Brown DW (2004) Rubella. Lancet 363: 1127-1137.

3. CDC (2011) Epidemiology and Prevention of Vaccine-Preventable Diseases (12 ${ }^{\text {th }}$ edn). The Pink Book 279-290.

4. Rodriguez R, Herrero B, Bartha JL (2013) The continuing enigma of the fetal echogenic intracardiac focus in prenatal ultrasound. Curr Opin Obstet Gynecol 25: 145-151.
5. Hutton J, Rowan P, Greisinger A, Mouzoon M4 (2014) Rubella monitoring in pregnancy as a means for evaluating a possible reemergence of rubella. Am J Obstet Gynecol .

6. Rorke LB (1973) Nervous system lesions in the congenital rubella syndrome. Arch Otolaryngol 98: 249-251.

7. Lane B, Sullivan EV, Lim KO, Beal DM, Harvey RL Jr, et al. (1996) White matter MR hyperintensities in adult patients with congenital rubella. AJNR Am J Neuroradiol 17: 99-103.

8. Chess S (1971) Autism in children with congenital rubella. J Autism Child Schizophr 1: 33-47.

9. Chess S (1977) Follow-up report on autism in congenital rubella. J Autism Child Schizophr 7: 69-81. 